\title{
Populus spp., respuestas clonales de crecimiento en suelos Argiudoles y Hapludoles, de la Provincia de Buenos Aires, Argentina
}

\author{
Esteban Baridón ${ }^{1}$, Mario Flores Palenzona² ${ }^{2}$ Raúl Marlats ${ }^{1}$, \\ Gabriela Senisterra $^{1}$ y Guillermo Millán ${ }^{1}$
}

${ }^{1}$ Departamento Ambiente y Recursos Naturales, Facultad de Ciencias Agrarias y Forestales, Universidad Nacional de La Plata. Calle 60 y 119 s/n (1900), La Plata, Argentina. ${ }^{2}$ Secretaria de Agricultura, Ganadería, Pesca y Alimentación de la Nación, Av. Paseo Colón 982, Buenos Aires, Argentina.

\begin{abstract}
E. Baridón, M. Flores Palenzona, R. Marlats, G. Senisterra, and G. Millán. 2008. Populus spp. clonal response of growth in Argiudoll and Hapludoll soils in Buenos Aires Province, Argentina. Cien. Inv. Agr. 35(3):287-292. The aim of this study was to assess the growth of clones of Populus spp. in the soil types Hapludolls and Argiudolls, of the mainland in the Buenos Aires province. The study took place in Alberti (3450' S, 60³0' W, 35 m.a.s.1.), Buenos Aires, Argentina. Five clones of Populus spp. were studied: 'Stoneville 66' ('Delta Gold'); 'Conti 12', '568-1', '610-31' and '564-17'. The experimental design consisted of randomized complete blocks with sub-samples. There were three blocks on three types of soil: a Typic Hapludoll, Entic Hapludoll, and a Typic Argiudoll. Physical and chemical characterizations of each soil type were performed. The average values of total basal area and height were recorded. The results were subjected to analysis of variance, and means were separated according to Tukey's test. An analysis of genotype-soil interaction was performed. No significant interaction between clone factor and soil factor was obtained. Clones 'Stoneville 66', and '568-1' did not differ significantly among themselves and displayed better growth. Therefore, no specific associations between these clones and the soils existed. These results should be valid and applicable for a large amount of land because of the wide distribution of Argiudoll and Hapludoll soils.
\end{abstract}

Key words: Argentina, Argiudolls, Hapludolls, poplar growth, Populus spp. Salicaceae.

\section{Introducción}

Las salicáceas (Familia Salicaceae) representan el tercer cultivo forestal de importancia en Argentina con 75.000 ha plantadas, equivalente al $12 \%$ de la superficie forestal. Su producción se concentra en las regiones del delta del Río Paraná, Pampa húmeda y zonas de regadío de Cuyo y Río Negro. Importantes diferencias ambientales existen entre estas zonas goegraficas. La zona delta del Río Paraná, con aproximadamente 40.000 ha de álamos (Populus spp.) y sauces (Salix spp.), es la de zona de mayor producción forestal debido a la aptitud productiva de los suelos y

Recibido 21 diciembre 2007. Aceptado 07 julio 2008

${ }^{1}$ Dirigir correspondencia a E. Baridón: jbaridon@agro.unlp.edu.ar a la proximidad a industrias transformadoras (Achinelli et al., 2004).

Según el censo agropecuario de 2002, el 48\% de las plantaciones de salicáceas en provincia de Buenos Aires correspondieron a álamos. Las periódicas inundaciones que afectan esta zona, origina demoras y/o interrupciones en el suministro de madera a las industrias. Esto ha motivado un incremento de nuevas plantaciones, fundamentalmente Populus spp., en la región Pampa húmeda para asegurar la continuidad en el abastecimiento de madera. Dichas plantaciones están distribuidas en el centro-norte de Buenos Aires y en el sur de Santa Fe. Se encuentran principalmente sobre suelos de aptitud de uso agrícola, Clase I, del tipo Argiudoles y Hapludoles, en zonas con precipitaciones medias anuales de 700 a 1000 mm (INTA, 1992). Los 
elevados rendimientos volumétricos de madera obtenidos en la región, podrían favorecer el cultivo de álamos como complemento de las actividades agropecuarias (Marlats et al., 1999). No obstante, la importancia de las plantaciones en suelos libres de inundaciones radica en su rol de reservas estratégicas de madera para suministrar madera como resguardo de las inundaciones del delta del Paraná. Achinelli et al. (2004) hacen referencia a que las 5.000 ha de cultivo de salicáceas existentes en la Pampa húmeda, se podrían expandir a 4 millones de ha. Esta situación sería perfectamente posible considerando que los suelos Argiudoles típicos y los Hapludoles típicos abarcan aproximadamente 12 millones de ha en la Pampa húmeda argentina (Godagnone et al., 2002).

El análisis de distintos clones de Populus ha demostrado alta variabilidad fenológica, fisiológica y diferencias en crecimiento, propiedades de la madera, y en el arraigamiento (Marlats et al., 2004). Asimismo, se han registrado interacciones significativas entre clones y sitios de plantación para los caracteres anteriormente mencionados (Senisterra et $a l ., 2000)$. Se han reportado diferencias en el comportamiento entre clones y sitios de cultivo, así como interacciones significativas entre clones y sitios de cultivo (Yu y Pulkkinen, 2003; Tomas et al., 1997 y Liu y Dickmann, 1996). Por ejemplo, diferencias significativas de crecimiento en altura y diámetro basal se han reportado para 25 clones de Populus en cuatro lugares con diferentes tipo e historial de uso del suelo, existiendo interacciones significativas entre el sitio de cultivo y los clones para las variables estudiadas (Yu y Pulkkinen, 2003).

Se ha demostrado, a nivel de micrositios, efectos significativos del contenido y tensión del agua edáfica sobre el crecimiento en los primeros años de plantaciones de Populus sp. (Baridón et al., 2004).

En la variabilidad del comportamiento clonal de especies de Populus, se destaca que la mayoría de los clones plantados en la zona continental fueron seleccionados para la región del delta del Río Paraná (Ragonese, 1987). Consecuentemente, existe un supuesto genético forestal de comportamiento azaroso para los nuevos sitios, ya que, los genotipos empleados en la región de la Pampa ondulada provienen de otros lugares de cultivo. Su elección fue empírica o del resultado de algunos pocos ensayos. Por lo tanto, existe la necesidad de estudiar la adaptación del material existente, del material a introducir y establecer las estrategias destinadas a la creación de clones para las nuevas zonas de plantación.

Por lo tanto, la expansión del cultivo de Populus spp. requeriría de la evaluación del material genético en uso y de nuevos genotipos para cada situación edafoclimática. Sólo así sería posible mejorar los rendimientos y la calidad. En consecuencia, se postula que el material genotípico disponible para la zona continental de la Provincia de Buenos Aires es crítico para extender el área de producción de álamo. El objetivo del presente trabajo fue evaluar el crecimiento de cinco clones de Populus spp. en suelos del tipo Hapludoles y Argiudoles presentes en la zona continental de la Provincia de Buenos Aires.

\section{Materiales y métodos}

\section{Localidad}

El estudio se realizó en un establecimiento forestal de Papel Prensa S.A., Alberti (34 ${ }^{\circ} 50^{\prime}$ S, 60³0' O, 35 m.s.n.m.), provincia de Buenos Aires, Argentina.

\section{Material vegetal}

Se evaluaron cinco clones, en estado de pruebas de productividad en la zona del desarrollo del presente trabajo pero de amplio uso en la región del delta del Río Paraná. Estos clones correspondierona Populus deltoides: 'Stoneville 66' ('Delta Gold'), 'A-610-31' y 'A-564-17' y a $P$. x canadensis: 'I-Conti 12' y 'A-568-1'. El material de plantación fue seleccionado y homogeneizado por topófisis y diámetro medio, utilizando estacas de aproximadamente $30 \mathrm{~cm}$ de longitud.

\section{Caracterización del suelo}

Los suelos se caracterizaron por cartografía, fotografías aéreas y por examen directo en el 
campo. Se efectuó la descripción morfológica de perfiles representativos. En cada suelo, se tomó una muestra por horizonte hasta 150 $\mathrm{cm}$ de profundidad, las que se caracterizaron respecto del contenido de carbono orgánico (Walkley Black), nitrógeno total (Kjeldahl), composición granulométrica (Bouyoucus), humedad (gravimétrico), $\mathrm{pH}$ (potenciométrico en relación suelo-agua 1:2,5), capacidad de intercambio catiónico (CIC) (método del acetato de amonio, $1 \mathrm{~N}, \mathrm{pH}$ 7); contenido de calcio y magnesio (volumetría con EDTA), sodio y potasio (fotometría de llama) y conductividad eléctrica (SSSA Book, 1996). Se calculó la suma de bases, saturación de bases, porcentaje de sodio intercambiable (PSI) y relación carbono nitrógeno $(\mathrm{C} / \mathrm{N})$.

\section{Evaluación}

Las mediciones se realizaron al finalizar la cuarta estación de crecimiento. Se determinó la altura total media (ATM) con un hipsómetro (Blume Leiss de $0,5 \mathrm{~m}$ de precisión) y el diámetro del tronco a la altura del pecho (DAP), con una forcípula $(0.01 \mathrm{~cm}$ de precisión). $\mathrm{Se}$ calculó el área basal media (ABM).

\section{Diseño y análisis estadísticos}

El área experimental tuvo $4.880 \mathrm{~m}^{2}$, plantadas a 3.5 x $2.8 \mathrm{~m}$, equivalente a 1.020 planta $^{-1} \mathrm{ha}^{-1}$. El diseño experimental fue de bloques completos al azar con submuestreo. Se instaló un bloque experimental en cada uno de los siguientes tipos de suelo: Hapludol típico, Hapludol éntico y Argiudol típico (NRCS, 2003). Los bloques experimentales se aislaron mediante una doble hilera perimetral de borde, conformada por estacas de P. $x$ canadensis 'Conti 12'. En cada bloque experimental se distribuyeron al azar 18 parcelas por cada clon de un árbol cada una. Se realizaron dos mediciones sobre la unidad experimental completa. Los resultados fueron evaluados por análisis de la varianza (ANDEVA), y los promedios se separaron de acuerdo con la prueba de comparación múltiple de Tukey $(\mathrm{p} \leq 0,05)$.

\section{Resultados y discusión}

La caracterización de los suelos de los tres bloques del ensayo permitió establecer la ausencia de limitaciones físico-químicas para el buen crecimiento radical (Cuadro 1). No obstante, hubo diferencias en textura, con un mayor contenido de arena en todos los horizontes del Hapludol éntico (Cuadro 1). Esto condiciona el almacenamiento de agua y la fertilidad potencial expresada a través de los valores de CIC y bases de cambio. Previamente, Liu y Dickmann (1996) señalaron la existencia de interacciones significativas entre clones de Populus y fertilidad y estrés hídrico. En forma similar, Baridón et al. (2004) comprobaron la existencia de efectos significativos del contenido y tensión del agua edáfica, sobre el crecimiento de Populus $s p$ en los primeros años de posplantación.

Con referencia a las mediciones dasométricas, Bratovich et al. (1996) determinaron que en salicáceas, el 40-50\% del turno de corta (rotación), sería tiempo suficiente para pronosticar significativamente los crecimientos a la cosecha. Considerando turnos de 10 años (de uso común en árboles para la industria celulósica) la evaluación de este trabajo a los 4 años de edad generó resultados que permiten establecer la tendencia del comportamiento final de los clones.

Debido a la mayor sensibilidad en la diferenciación del vigor de distintos clones de álamos, Padró Simarro (1982) sugirió el uso del ABM y ATM. En forma similar, Maltano et al., (1995) señalaron que el análisis comparativo de las ABMs, al enfatizar las tendencias de los diámetros, resultó útil para identificar las fracciones económicamente más valiosas de cada tratamiento. Sobre la base de lo expuesto, en este trabajo se utilizó la ATM y el ABM como parámetros de comparación para el crecimiento clonal en los distintos suelos empleados en este estudio.

Las ATMs registradas en los clones '568-1' y 'Stoneville 66' no presentaron diferencias significativas en los suelos Argiudol típico y Hapludol típico. En el suelo Hapludol éntico se manifestó un comportamiento diferencial, destacándose el crecimiento en altura del clon 'Stoneville 66' con respecto a los restantes clones evaluados (Figura 1). 
Cuadro 1. Descripción analítica de los suelos Hapludol y Arguidol empleados en este trabajo para la evaluación de clones de Populus spp.

Table 1. Analytical description of the Hapludoll and Arguidoll soils used to evaluate clones of Populus spp. in this study.

\begin{tabular}{|c|c|c|c|c|c|c|c|c|c|c|c|}
\hline \multirow[t]{2}{*}{ Características del suelo } & \multicolumn{3}{|c|}{$\begin{array}{l}\text { Hapludol éntico } \\
\text { Horizonte }\end{array}$} & \multicolumn{4}{|c|}{$\begin{array}{l}\text { Hapludol típico } \\
\text { Horizonte }\end{array}$} & \multicolumn{4}{|c|}{$\begin{array}{l}\text { Argiudol típico } \\
\text { Horizonte }\end{array}$} \\
\hline & Ap & $\mathrm{AC}$ & $\mathrm{C}$ & Ap & $\mathrm{Bw}$ & $\mathrm{BC}$ & $\mathrm{C}$ & Ap & $\mathrm{Bt}$ & $\mathrm{BC}$ & $\mathrm{C}$ \\
\hline Profundidad, $\mathrm{cm}$ & $0-25$ & $25-40$ & $>40$ & $0-17$ & $17-45$ & $45-70$ & $>70$ & $0-25$ & $25-66$ & $66-90$ & $>90$ \\
\hline Materia orgánica, $\%$ & 2,10 & 0,90 & -2 & 2,00 & 2,20 & 1,30 & -2 & 3,09 & 1,65 & 0,77 & -2 \\
\hline Carbono total $(\mathrm{C}), \%$ & 1,20 & 0,50 & - & 1,20 & 1,30 & 0,70 & - & 1,82 & 0,97 & 0,44 & - \\
\hline Nitrógeno total (N), \% & 0,10 & 0,05 & - & 0,10 & 0,10 & 0,10 & - & 0,16 & 0,10 & 0,07 & - \\
\hline Relación C/N & 12,00 & 10,00 & - & 9,90 & 11,30 & 9,50 & - & 11,38 & 9,80 & 6,67 & - \\
\hline Arcilla $<2 \mu \mathrm{m}, \%$ & 10,70 & 2,70 & 1,02 & 17,60 & 19,60 & 19,60 & 19,60 & 19,50 & 25,50 & 16,80 & 6,70 \\
\hline Limo $2-50 \mu \mathrm{m}, \%$ & 20,00 & 26,00 & 24,00 & 32,00 & 28,00 & 26,00 & 24,00 & 42,00 & 33,90 & 38,00 & 34,80 \\
\hline Arena, $\%$ & 69,30 & 71,30 & 73,30 & 50,40 & 52,40 & 54,40 & 56,40 & 38,50 & 40,60 & 54,80 & 58,50 \\
\hline $\mathrm{pH}(1: 2,5)^{1}$ & 5,60 & 6,10 & 6,30 & 6,60 & 6,60 & 6,70 & 6,70 & 5,65 & 6,20 & 6,50 & 7,40 \\
\hline \multicolumn{12}{|c|}{ Cationes de intercambio, $\mathrm{cmol} \cdot \mathrm{kg}^{-1}$} \\
\hline $\mathrm{Ca}++$ & 8,00 & 6,00 & 6,50 & 8,60 & 7,20 & 7,00 & 7,40 & 11,50 & 11,00 & 10,00 & 7,80 \\
\hline $\mathrm{Mg}++$ & 2,00 & 2,90 & 2,50 & 1,90 & 2,30 & 2,70 & 3,20 & 2,45 & 3,40 & 2,70 & 2,70 \\
\hline $\mathrm{Na}+$ & 0,20 & 0,60 & 0,40 & 0,40 & 0,30 & 0,50 & 0,50 & 0,15 & 0,20 & 0,20 & 0,20 \\
\hline $\mathrm{K}+$ & 1,20 & 1,40 & 1,30 & 1,80 & 1,60 & 1,60 & 0,90 & 1,75 & 1,10 & 0,80 & 1,20 \\
\hline Suma de bases, $\mathrm{cmol} \cdot \mathrm{kg}^{-1}$ & 11,50 & 10,90 & 10,70 & 12,70 & 11,40 & 11,80 & 12,00 & 15,85 & 15,70 & 13,70 & 11,90 \\
\hline $\mathrm{CIC}, \mathrm{cmol} \cdot \mathrm{kg}^{-1}$ & 12,90 & 10,90 & 11,50 & 13,80 & 12,00 & 13,30 & 13,30 & 19,50 & 18,40 & 16,30 & 13,70 \\
\hline Saturación de bases, $\%$ & 88,30 & 100,00 & 93,00 & 91,60 & 95,90 & 88,90 & 91,00 & 81,28 & 85,33 & 84,05 & 86,86 \\
\hline $\begin{array}{l}\text { Sodio de intercambio, } \\
\text { (PSI), \% }\end{array}$ & 1,50 & 5,50 & 3,50 & 2,90 & 2,90 & 3,80 & 3,80 & 0,77 & 1,09 & 1,23 & 1,46 \\
\hline
\end{tabular}

${ }^{1} \mathrm{pH}$ determinado en un extracto de suelo en proporción 1:2,5; suelo:agua.

2 -, valor no determinado.

${ }^{1} \mathrm{pH}$ determined in a 1:2.5 (soil:water) soil extract.

${ }_{2}-$, not determined.

Existieron considerables diferencias entre clones respecto de las $\mathrm{ABM}$ obtenidos en cada bloque experimental (Figura 1B). Según el ANDEVA hubo diferencias significativas ( $\mathrm{p} \leq 0,05)$ en los tres suelos empleados en este trabajo. El clon 'Stoneville 66' presentó el mayor crecimiento en Hapludol éntico, mientras que en el Hapludol típico y en el Argiudol típico las ABM superiores correspondieron al clon '568-1' de $P$. $x$ canadienses. Estos resultados coincidieron con los previamente reportados por Senisterra et al. (2000) en árboles de 5 años. Según estos autores, el crecimiento medio superior del clon '568-1' se observaría a partir del tercer año. El mayor desarrollo del clon '568-1' con respecto a otros clones se podría deber a una más prolongada estación de crecimiento (Gennari et al. (2004).

Las diferencias de crecimiento entre clones fueron significativas $(\mathrm{p} \leq 0,05)$. En forma análoga también fueron significativas las diferencias de ABM entre los distintos suelos (bloques experimentales), independientemente de los clones evaluados. La interacción entre el clon y el tipo de suelo no fue significativa (Cuadro 2). Estos resultados difieren de reportes previos donde se reportan interacciones significativas entre suelos y clones (Tomas et al., 1997; Yu y Pulkkinen, 2003). No obstante, se debe considerar que Yu y Pulkkinen (2003) trabajaron con genotipos de Populus distintos a los ensayados en el presente trabajo, en cuatro suelos marcadamente diferentes y con historias agrícolas distintas, lo que en alguna medida confirma la individualidad de las respuestas obtenidas.

Aunque se acepta la existencia de diferenciales de crecimiento entre los clones para ambientes distintos, el material clonal plantado actualmente en la parte continental, provino de trabajos de mejoramiento genético destinados a satisfacer las necesidades de la región del delta del Río Paraná (Ragonese, 1987). Además se empleó material vegetal cuyos criterios de elección no fueron debidamente definidos para estos nuevos sitios de cultivo de Populus spp. No obstante, la sobrevivencia, sanidad y crecimiento han sido 

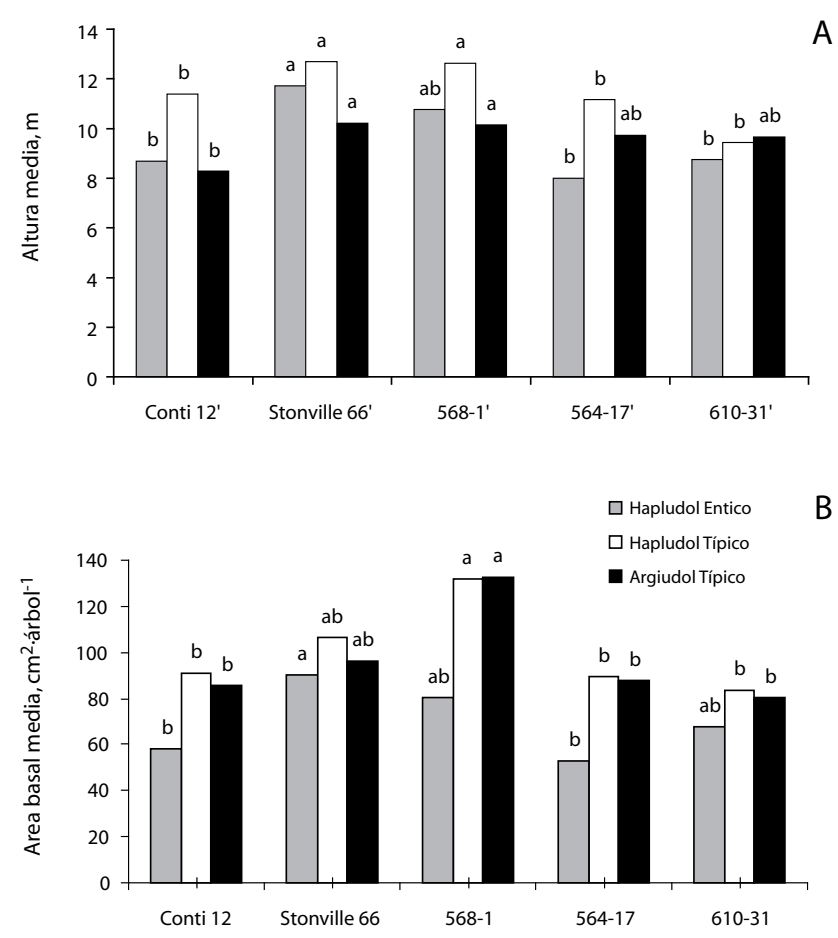

Figura 1. Caracterización de clones de álamos (Populus spp.) determinada por clon y tipo de suelo, determinada en árboles de cuatro años de edad. A. Altura media B. Area media basal. Promedios seguidos por iguales letras no son estadísticamente diferentes entre sí según la prueba de comparación múltiple de Tukey $(\mathrm{p}=0,05)$.

Figure 1. Characterization of poplar (Populus spp.) clones, by clone and soil type, in 4-year-old trees. A. Mean height. B. Mean basal area. Means followed by the same letters are not statistically different according to Tukey's multiple range comparison test $(p=0.05)$.

aceptables, con un rango de incremento medio anual de 14 a 18 tha" ${ }^{-1} \cdot a_{n} o^{-1}$ (Achinelli et al., 2004).

Al cuarto año de su establecimiento, los clones 'Stoneville 66' y '568-1' tuvieron el mejor comportamiento en los suelos de los lugares de ensayo. El crecimiento, caracterizado a través del área basal media, demostró que éstos clones son potencialmente superiores respecto de los restantes clones incluidos en este trabajo. Independientemente del sitio de cultivo, 'Stoneville 66' y '568-1' fueron estadísticamente similares. Por lo que no existirían asociaciones específicas entre estos clones y los suelos en los cuales se cultivaron. No obstante, 'Stoneville 66' mostró una tendencia a alcanzar mejores crecimientos en suelos Hapludoles énticos. Estos resultados confirman la necesidad de investigaciones adicionales destinadas a establecer clones de Populus spp. para favorecer la actividad forestal en la región. Los resultados obtenidos son válidos dentro de la unidad climática considerada en este trabajo que incluye una importante superficie de suelo representada por Hapludoles típicos y énticos, y en menor medida Argiudoles típicos.

\section{Resumen}

El objetivo del presente trabajo fue evaluar el crecimiento de clones de Populus spp. en suelos de tipo Hapludoles y Argiudoles de la zona continental de la Provincia de Buenos Aires. El estudio se localizó en Alberti (3450' S, 60³0' O, 35 m.s.n.m.), Buenos Aires, Argentina y se utilizó Populus deltoides ('Stoneville 66' ('Delta Gold'), 'A-610-31' y 'A-564-17') y $P$. $\mathrm{x}$ canadensis ('I-Conti 12' y 'A-568-1'). El diseño experimental fue de bloques completos al azar con submuestreo. Se instalaron tres 
bloques, sobre tres tipos de suelo: Hapludol típico, Hapludol éntico y Argiudol típico. Se realizó una caracterización físico-química de cada suelo, se registraron los valores medios de área basal y altura total de los árboles. Los resultados se sometieron a análisis de la varianza y los promedios se separaron según Tukey ( $p$ $\leq 0,05)$. Se realizó un análisis de interacción genotipo-suelo que no arrojó interacciones significativas. Los clones 'Stoneville 66' y '568-1' tuvieron el mejor crecimiento, sin diferencias significativas entre sí, por lo que no existirían asociaciones específicas entre esos clones y los suelos considerados. Los resultados obtenidos en este estudio serían válidos para una importante superficie de tierras debido a la amplia distribución areal de los suelos Argiudoles y Hapludoles dentro de la unidad climática considerada.

Palabras clave: Argentina, Argiudoles, crecimiento, Hapludoles, Populus spp., Salicaceae.

\section{Literatura citada}

Achinelli, F., G. Denegri y M. Marlats. 2004. Evolución y perspectivas del cultivo de salicáceas en la pampa húmeda argentina. SAGP y A Forestal (Argentina) 32:14-23.

Baridón, E., A. Pellegrini, V. Cattani y F. Achinelli. 2004. Efectos del agua edáfica sobre los primeros años de implantación de clones de Populus sp: tres situaciones de micro-relieve en Hapludoles Típicos. En: X Reunión Argentina y IV Latinoaméricana de Agrometeorología. Mar del Plata, Buenos Aires, Argentina. 2 pp.

Bratovich, R., R.M. Marlats y H. Mikelaites. 1996. Relación juvenil-adulto de crecimientos en alturas, diámetros y volúmenes de clones provenientes de cruzamientos controlados inter e intraespecíficos de Populus sp. L. Revista de la Facultad de Ciencias Agrarias y Forestales (Argentina).1:7-13.

Gennari, A., E. Prada, F. Achinelli y P. Vivas. 2004. Manejo del material de plantación para mejorar la supervivencia en clones de Populus deltoides Bartr. ex Marsh. obtenidos en la Argentina. Actas de la $22^{\circ}$ Reunión de la Comisión Internacional del Alamo IPC-2004, Santiago, Chile, 98 pp.

Godagnone, R.E., H. Bertola y M. Ancarola. 2002. Mapa de suelos de la Argentina. Instituto Geográfico Militar. Buenos Aires, Argentina.

INTA. 1992. Cartas de Suelos de la República Argentina. Hoja 3560-21 (Bragado). Instituto
Nacional de Tecnología Agropecuaria (INTA). Buenos Aires, Argentina. 91 pp.

Liu, Z.J. y D.I. Dickmann. 1996. Effects of water and nitrogen interaction on net photosynthesis, stomatal conductance and water use efficiency in two hybrid poplar clones. Phys. Plant. 97:507-512.

Maltano, M., J. Puumalainen y R. Päivinen. 1995. Comparison of Beta and Weibull functions for modelling basal area diameter distribution in stand of Pinus sylvestris and Pices abies. Scandinavian Journal Forestry Res. 10:284-295.

Marlats, R.M., G.E. Senisterra, J.W. Lanfranco, J.L. Marquina y M.E Vazquez. 2004. Populus spp: Estabilidad y ganancia genética sobre la altura media dominante en tres ambientes de la pampa ondulada, Buenos Aires, Argentina. Revista de la Facultad de Ciencias Agrarias, Universidad Nacional de Cuyo (Argentina) 36:9-16.

Marlats, R.M., J.L. Marquina y J.W. Lanfranco. 1999. Populus deltoides Marsh: comportamiento clonal en relación a parámetros de clima y suelo en la zona continental de la provincia de Buenos Aires. Páginas 44-49. En: Actas del $14^{\circ}$ Congreso Latinoamericano de la Ciencia del Suelo. Departamento de Ciencias Químicas, Universidad de la Frontera, Temuco, Chile.

NRCS. 2003. Keys to Soil Taxonomy. Ninth Ed. Natural Resources Conservation Service (NRCS).United State Department of Agriculture (USDA). Washingon D.C.,USA.332 pp.

Padró Simarro, A. 1992. Clones de Chopo para el valle medio del Ebro. Servicio de Investigación Agraria, Diputación General de Aragón. Zaragoza, España. 203 pp.

Ragonese, A.E. 1987. Fitotecnia de Salicáceas en el Centro Nacional de Investigaciones Agropecuarias (INTA). Comunicación del Académico de Número, Sesión Ordinario de la Academia Nacional de Agronomía y Veterinaria. Tomo XLI, 6: 30 pp.

Senisterra, G., R.M. Marlats, M. Vázquez, J.W. Lanfranco y J.L. Marquina. 2000. Comportamiento de clones de álamos (Populus spp.) implantados en dos sitios de la pampa húmeda, Argentina. Revista Forestal YVYRARETA 10:66-73.

SSSA. 1996. Book Series: 5. Methods of Soil Analysis. Part 3. Chemical Methods. Published by Soil Science Society of America (SSSA), American Society of Agronomy. Madison, Wisconsin, USA. $497 \mathrm{pp}$.

Thomas, B.R., S.E. Macdonald y B.P. Dancik. 1997. Variance components, heritabilities and gain estimates for growth chamber and field performance of Populus tremuloides: growth parameters. Silvae Genet. 46:317-326.

Yu, Q. y P. Pulkkinen. 2003. Genotype-environment interaction and stability in growth of aspen hybrid clones. Forest Ecology and Mangement. 173:25-35. 